expression of TSLP and its receptor (TSLP-R) in the duodenal mucosa of patients affected by RCD.

Methods Duodenal biopsies were collected from 12 RCD patients, 16 uncomplicated CD patients before and after 12 months of gluten-free diet, and 14 control subjects. The gene expression of TSLP and TSLP-R was evaluated on biopsy homogenates by quantitative RT-PCR, and the data were normalised for cytokeratin 18 expression. The protein expression of TSLP and TSLP-R was studied on biopsy homogenates by immunoprecipitation and on biopsy sections by confocal microscopy.

Results In vivo mucosal TSLP expression was significantly reduced both at the mRNA and protein levels in the duodenum of RCD and untreated CD patients compared to treated CD patients and controls, without differences between RCD and untreated $\mathrm{CD}$ patients and between treated $\mathrm{CD}$ patients and controls. TSLP transcript down-regulation in untreated CD mucosa was confirmed after normalisation for cytokeratin 18. TSLP-R was expressed in the duodenal mucosa both at the gene and the protein level, without significant differences between RCD, untreated and treated CD patients and control subjects. Confocal microscopy analysis confirmed these findings.

Conclusion TSLP expression is primarily reduced in the duodenal mucosa of RCD patients. Further studies are needed to clarify the influence of TSLP reduction on the process of immunosurveillance in RCD.

Disclosure of Interest None Declared.

\section{PTH-118 ADHERENCE TO DUODENAL BIOPSY GUIDELINES INCREASES THE DETECTION OF COELIAC DISEASE: A MULTICENTRE UK STUDY}

${ }^{1} \mathrm{PD}$ Mooney ${ }^{*},{ }^{2} \mathrm{M}$ Finegan, ${ }^{3} \mathrm{~F}$ Khan, ${ }^{2} \mathrm{R}$ Keld, ${ }^{3} \mathrm{G}$ Naylor, ${ }^{1} \mathrm{DS}$ Sanders. ${ }^{1}$ Regional $\mathrm{Gl}$ and Liver Unit, Royal Hallamshire Hospital, Sheffield, UK; ${ }^{2}$ Gastroenterology, Royal Edward Albert Infirmary, Wigan, UK; ${ }^{3}$ Gastroenterology, Chesterfield Royal Hospital, Chesterfield, UK

\subsection{6/gutjpl-2014-307263.564}

Introduction Coeliac disease (CD) is a common autoimmune condition affecting $1 \%$ of the adult population. However large numbers of patients remain undiagnosed which may have significant health consequences. Guidelines suggest that at least 4 duodenal biopsies should be taken to rule out CD. A previous US study showed that biopsy guidelines were only followed in 35\% of cases. The aim of the present study was to see whether guidelines were being followed in the UK and if adherence to the guidelines improved detection of $\mathrm{CD}$.

Methods Endoscopy and histology reports were retrospectively reviewed for all patients who had a duodenal biopsy in a 3 month period between November 2012 and January 2013 from 4 UK hospitals. Indications for biopsy, role of the endoscopist, number of duodenal biopsies received by histopathology and the final diagnosis were recorded. The presence of villous atrophy was required for CD diagnosis. Patients were excluded if they had known CD. The difference between a double and single bite biopsy technique was also assessed.

Results 1423 patients underwent duodenal biopsy for possible CD across the 4 sites in the study period. 97 (6.8\%) of these were diagnosed with $\mathrm{CD}$. Guidelines to take at least 4 biopsies were met in $40 \%$ of patients and the median number of duodenal biopsies taken for all patients was 3. CD diagnosis was more likely guidelines were followed $(10.1$ vs. $4.6 \% \mathrm{p}<0.0001)$. The median number of biopsies was greater in patients diagnosed with CD (4 vs. 3) $\mathrm{p}<0.0001$. Gastroenterologists and nurse endoscopists were more likely than surgeons to follow guidelines $(41.8 \%$ vs $51.2 \%$ vs $18.2 \% \mathrm{p}<0.0001)$ and took a higher median number of biopsies ( 3 vs. 4 vs. 2 p $<0.0001$ ). As a result gastroenterologists and nurse endoscopists made a diagnosis of $\mathrm{CD}$ in more cases than surgeons $(7.1$ vs. $6.7 \%$ vs. $3.0 \% \mathrm{p}$ 0.1). All presenting characteristics (other than positive serology in which guidelines were followed in 65\%) were associated with poor adherence to guidelines. $12.4 \%$ of newly diagnosed CD patients had at least 1 non-diagnostic gastroscopy in the 5 years prior to diagnosis. Changing biopsy technique to single bites resulted in improvement of median D2 biopsies from 3 to 4 . ( $p$ $0.02)$.

Conclusion We have shown that $12.4 \%$ of patients with CD had a previous gastroscopy 5 years prior to their diagnosis. Taking 4 duodenal biopsies results in increased detection of CD. We are the first investigators to demonstrate variation in biopsy rates based on the speciality of the endoscopist and biopsy technique. Furthermore this variability has a direct relationship with the detection rate of CD. Education of all groups of clinicians in duodenal biopsy techniques may result in more patients receiving a prompt diagnosis of $\mathrm{CD}$.

Disclosure of Interest None Declared.

\section{PTH-119 HIGH DEFINITION (HD) ENDOSCOPY WITH I-SCAN FOR THE DETECTION OF MARKERS OF COELIAC DISEASE: A FEASIBILITY STUDY}

PD Mooney*, S Wong, A Johnston, DS Sanders. Regional Gl and Liver Unit, Royal Hallamshire Hospital, Sheffield, UK

\subsection{6/gutjnl-2014-307263.565}

Introduction Coeliac disease (CD) remains underdiagnosed. Previous studies have shown that up to $13 \%$ of patients with CD have undergone a previous gastroscopy where the opportunity to take duodenal biopsies and make a diagnosis had been missed. Clinicians may rely on the presence of endoscopic markers of $\mathrm{CD}$ to guide biopsy however these have been shown to lack the required sensitivity. A routine duodenal biopsy approach may solve this problem but this is time consuming and expensive. Methods to improve the macroscopic detection of $\mathrm{CD}$ at endoscopy to guide biopsy would seem advantageous. A single trial on I-Scan, a commercially available digital enhancement technique, has shown promising results in identifying markers of villous atrophy. However this was an uncontrolled, unblinded trial in high prevalence population ( $35 \% \mathrm{CD})$. We aimed to assess the utility of I-Scan in a lower prevalence population in a randomised controlled trial.

Methods Patients on a single coeliac enriched endoscopy list were randomised into 2 groups. Group 1 standard HD white light endoscopy (WLE) and group 2 WLE plus I-Scan. The presence of endoscopic markers of CD, scalloping, mosaic pattern, nodularity, loss of duodenal folds or increased vascularity was noted throughout the duodenum. All patients received 4 biopsies from the second part of the duodenum and at least 1 biopsy from the bulb. Coeliac serology was taken at the time of endoscopy. Macroscopic markers of $\mathrm{CD}$ are compared to the presence of villous atrophy on histology as the gold standard. 3, 10-point likert scales for pain, discomfort and distress were used to assess tolerability. 
Results 116 patients (66 female, mean age 54.9 SD 17.5) have been recruited to date (55 into group 1 and 61 in group 2). In total $14(12.1 \%)$ new diagnoses of CD have been made. I-Scan appears to enhance the appearance of markers for $\mathrm{CD}$ and in a single patient in group $2 \mathrm{CD}$ markers that were not noted to be seen on WLE became apparent. Preliminary results show that endoscopic markers of $\mathrm{CD}$ across both groups currently have a sensitivity of $78.6 \%$ (48.8 - 94.3), specificity $82.4 \%$ (73.3 $88.9)$, positive and negative predictive values of $37.9 \%(21.3-$ $57.6)$ and $96.6(89.5$ - 99.1). Median tolerability scores were good in both groups but better in the I-Scan group than WLE alone (4/30 vs. $8 / 30 \mathrm{p} 0.005)$.

Conclusion The addition of I-Scan to standard endoscopy to aid the diagnosis of $\mathrm{CD}$ is well tolerated and is feasible. I-Scan appears to enhance the markings of coeliac disease, however a larger study is required to truly evaluate the effectiveness of I-Scan as an adjunct to standard endoscopy to increase CD diagnosis.

Disclosure of Interest None Declared.

\section{PTH-120 COELIAC DISEASE AND DOUBLE-BALLOON ENTEROSCOPY: WHAT CAN WE ACHIEVE? THE EXPERIENCE OF TWO EUROPEAN TERTIARY REFERRAL CENTRES}

${ }^{1} \mathrm{PD}$ Mooney*, ${ }^{2} \mathrm{C}$ Tomba, ${ }^{2} \mathrm{~F}$ Branchi, ${ }^{1} \mathrm{DS}$ Sanders, ${ }^{1} \mathrm{R}$ Sidhu, ${ }^{2} \mathrm{M}$ Locatelli, ${ }^{2} \mathrm{D}$ Conte, ${ }^{2}$ MT Bardella, ${ }^{2} \mathrm{~L}$ Elli. ${ }^{1}$ Regional GI and Liver Unit, Royal Hallamshire Hospital, Sheffield, UK; ${ }^{2}$ Center for the Prevention and Diagnosis of Celiac Disease - Gastroenterology Unit II, Fondazione IRCCS Ca' Granda Ospedale Maggiore Policlinico, Milan, Italy

\subsection{6/gutjpl-2014-307263.566}

Introduction The indications for and efficacy of device-assisted enteroscopy is not standardised in coeliac disease (CD). We present the largest study to date to evaluate the clinical role of double-balloon enteroscopy (DBE) in complicated CD.

Methods DBE findings in CD patients with suspected small bowel complications were retrospectively evaluated in two tertiary referral centres (Milan and Sheffield). Demographic data of the studied cohort were compared with a database of 1000 non complicated CD patients.

Results Findings from 14 oral and seven anal DBE in 19 CD cases (11 males $\mathrm{p}=0.003$ vs control database) were reviewed. Mean age at $\mathrm{CD}$ diagnosis $(37 \pm 19$ vs. $27 \pm 18)$ and at small bowel evaluation $(49 \pm 15$ vs. $38 \pm 13)$ was significantly higher in the DBE group compared to controls ( $\mathrm{p}$ $<0.001$ ). Indications for DBE were the follow up of known refractory coeliac disease (RCD) (\#7), suspicion of small bowel complications due to gastrointestinal symptoms (\#4), severe iron deficiency anaemia (\#6) and long standing poor dietary adherence (\#2). All DBE were performed after small bowel capsule endoscopy, except for one case. 3 patients from the known RCD group had evidence of TCRg monoclonality on biopsy (type 2 RCD). One of these patients had jejunal ulceration whilst the other 2 cases had areas with small white raised patches. A further RCD case had evidence of jejunal ulceration however biopsies didn't show any evidence of TCRg monoclonality. A single RCD case had distally worsening atrophy. Patchy small bowel atrophy was observed in all the non adherent patients and in 2 patients with persistent gastrointestinal symptoms who had only been on a gluten free diet for a short time. Two jejunal adenocarcinomas and an ileal neuroendocrine tumour were detected. All 3 of these patients presented with iron deficiency anaemia.
Conclusion This is the largest international DBE outcomes study in CD patients. Even minor mucosal lesions seen at DBE may be associated with significant pathology. Evaluation of non-responsive/refractory symptoms by DBE was associated with older patients $(p<0.001)$ and a higher proportion of males $(p=0.003)$ than an uncomplicated CD population. Further studies are needed to better understand the clinical relevance of the small bowel endoscopic features and to optimise DBE indications.

Disclosure of Interest None Declared.

\section{PTH-121 THE BURDEN OF POOR NUTRITION IN CHRONIC PANCREATITIS: WHAT ARE THE IMPACT OF BEHAVIOURAL AND SOCIOECONOMIC FACTORS?}

${ }^{1} \mathrm{~B}$ Paranandi*, ${ }^{2} \mathrm{PS}$ Patel, ${ }^{1} \mathrm{GH}$ El-Sayed, 'D Joshi, ${ }^{3} \mathrm{~A}$ Ghai, ${ }^{3} \mathrm{~K}$ Koshy, ${ }^{1} \mathrm{MH}$ Chapman, ${ }^{1}$ SP Pereira, ${ }^{1} \mathrm{GJ}$ Webster, ${ }^{1} \mathrm{GJ} J$ Johnson. ${ }^{1}$ Pancreaticobiliary Medicine, University College London Hospitals, London, UK; ${ }^{2}$ Dietetics, University College London Hospitals, London, UK; ${ }^{3}$ UCL Medical School, London, UK

\subsection{6/gutjnl-2014-307263.567}

Introduction Nutrition centred research in pancreatitis has largely focused on acute pancreatitis. There is limited nutritional data in patients with Chronic Pancreatitis (CP), a condition that predisposes to endocrine and exocrine failure, fat-soluble vitamin deficiency and osteoporosis, which may worsen a patient's quality of life and long-term outcome. We aim to determine the prevalence of malnutrition (using the Malnutrition Universal Screening Tool -MUST), active alcohol consumption and cigarette smoking in patients with $\mathrm{CP}$.

Methods Prospective study of consecutive patients with CP attending a tertiary clinic between October and December 2013. They were invited to participate in a face-to-face questionnaire study. Behavioural and socioeconomic data were collated.

Results A cohort of 86 patients identified were predominantly male (67\%), White British (62\%), median age 58 years (range 18$90)$, of socio-economic class (SEC) 8 (21\% never worked/longterm unemployed) with educational level (EL) 1 (29\% degree or equivalent). Aetiologies included alcohol (29\%), idiopathic (25\%), autoimmune (22\%) and gallstones (11\%). The aetiology in 6\% was actively under investigation. Median follow up was 27.5 months (range $0-151$ ) from index appointment. Active alcohol exposure was noted in 33\% (28/86) with excessive amounts (m $>21 \mathrm{u}, \mathrm{f}>14 \mathrm{u} /$ week) in $8 \%(7 / 86)$. The majority of patients (> $70 \%$ ) with ongoing exposure to alcohol had primary aetiologies other than alcohol. Active cigarette smoking was noted in 34\%. MUST scores $\geq 1$ were noted in 38\% (33/86) conferring "medium to high risk of malnutrition". Multiple linear regression analysis of age, gender, ethnicity, SEC, EL, aetiology, alcohol exposure and smoking did not show any statistically significant variables that predicted MUST score. 27\% (9/33) patients "at risk of malnutrition" had been referred to or had been seen by a dietitian within the previous 12 months. The remaining 73\% (24/33) "at risk" patients all claimed to have received nil or suboptimal nutritional advice with regards to $\mathrm{CP}$ within the previous 24 months.

Conclusion Patients with CP attending our tertiary clinic are at significant risk of malnutrition, which may be under-recognised. Behavioural and socioeconomic factors do not allow reliable prediction of risk of malnutrition to be made, in this cohort. This data emphasises the need for an algorithmic approach to improve recognition of malnutrition in $\mathrm{CP}$ and for implementation of specialist dietetic, alcohol liaison and smoking cessation services within the outpatient environment.

Disclosure of Interest None Declared. 